\title{
Research on Higher Mathematics Teaching Based on Computer
}

\author{
Liu Zhonghua ${ }^{1, \text { a }}$ \\ ${ }^{1}$ Yishui Campus,Linyi University, Yishui Shandong , 276400, China \\ a81079684@qq.com
}

Key Words: computer and information technology; integration model; higher mathematics; teaching evaluation

\begin{abstract}
: this paper constructs information technology and teaching model of higher mathematics based on computer, it uses model of problem start-problem discussion-problem solution-reconsideration innovation to promote students to do automatic learning, enough course attraction, lively teaching all presents relatively abstract or complicated content in higher mathematic course. Through experiment it indicates that this model and experimental teaching model is favorable for students to accept the key points and difficult points in mathematics course, it is favorable to increase teaching quality of class, enhance learning enthusiasm and initiative of all the students, so it is very helpful for learning of higher mathematics.
\end{abstract}

\section{Introduction}

Higher mathematics not only plays an important position in the field of science and engineering discipline, but also it has penetrated into every field such as economy, finance as well as humanity and social science etc, it is increasingly becoming to be the important measure and tool ${ }^{[1,2]}$ for every curriculum to make scientific research, it has already become to be one curriculum with universal application value. As one kind of science, higher mathematics has its own characteristics, which has high abstraction, strict logic and universal application. By learning higher mathematics, it not only paves necessary base for students to learn subsequent mathematics curriculum and professional curriculum, but also can gradually cultivate the abstract thinking and logic thinking of students, enabling them to have relatively skilled computation ability and ability ${ }^{[3]}$ of comprehensively use learned knowledge to make analysis and further solve problems. Therefore, teaching quality of higher mathematics has important effect on learning quality in the whole university period, in addition, the learning process of higher mathematics also relates to science and cultural quality such as analysis thinking ability, logic recognition ability and potential creation etc. Higher mathematics undertakes the double function of basic education of science and culture; it is one of the important aspects of quality education for students. However, this kind of model is powerless for students to learn and master relatively abstract or complicated content. It is inevitable to fully apply advantages of network platform and computer multi-media to make research and explore the new integration model of information technology and higher mathematics course.

This paper tries to fully apply computer multi-media technology, software of computer aided instruction, it lively explains and presents relatively abstract or complicated content in higher mathematics, puts forward one kind of teaching model of mathematics test based on information technology. This kind of model aims at forming the subject consciousness of students, emphasizes at penetration of mathematics thinking model and personality development of students, making students go forward from close to open, encourage students to propose problems, reconsider problems and extend problems, enabling students to make new exploration during gradual enlargement process for students.

\section{Design on computer teaching of higher mathematics}

Whether it is learning motivation, aim, or learning environment and computation methods etc, all can stimulate interests of students and form subjective initiative by computer. The famous 
mathematics software is as follows: MATLAB, Mathematicia, Maple, SAS, SPSS etc are mainly used in statistics field. Lingo is expert in mathematics and number computation function(symbol computation function and graphics procession function all in one, and it is easily to learn and use, experience and exploration, the most effective language environment can provide each kind of mathematics tool to avoid complicated reduction and computation, save much more time and energy to learn and explore.

Integration of information technology and mathematics is to combine with subjective initiative and dominance of students, its core is to increase science quality of students, confirms multi-unit mathematics class teaching target from many aspects such as science knowledge and skill, science idea, science method, science attitude and science spirit etc by combining with the knowledge structure and ability base mastered by students, enabling students to gradually form scientific world view and methodology on basis of mastering basic knowledge and basic skill by students. In the process of teaching, we should fully use information and technology platform to lively develop key point teaching of higher mathematics curriculum, expand knowledge amount of higher mathematics, cultivate personalized learning of students and create conditions for students to actively participate in the whole process of mathematics class teaching by using computer and multi-media as Microsoft PowerPoint. As it is indicated by diagram 1, it is design model of computer platform for higher mathematics, it uses teaching model of Task-based learning to instruct integration of information technology and higher mathematics curriculum. Teaching method of Task-based Learning is one kind of methods to use student as center and task-driven as teaching model, it is the teaching method [5] established on teaching and theory base of constructivism. The learning activity of students should combine with relevant task and problem to guide and maintain learning interest and motivation of learners by completing task and solving problem, making students have task and problem to learn, students must have learning autonomy, so teachers should continually guide and encourage students. Teachers divide teaching content into many detailed tasks according to teaching topic, every task contains relevant knowledge and skill that should be mastered by students, guide students to analyze problem, propose methods and steps of solving problems through autonomous exploration and group cooperation, the process for students to complete task is the process for students to gradually find problems, analyze problems and solve problems. In the task-based learning, teachers are no longer dominate the whole teaching process, while they are only help students to complete tasks, teachers only plays the effect of illumination and instruction.

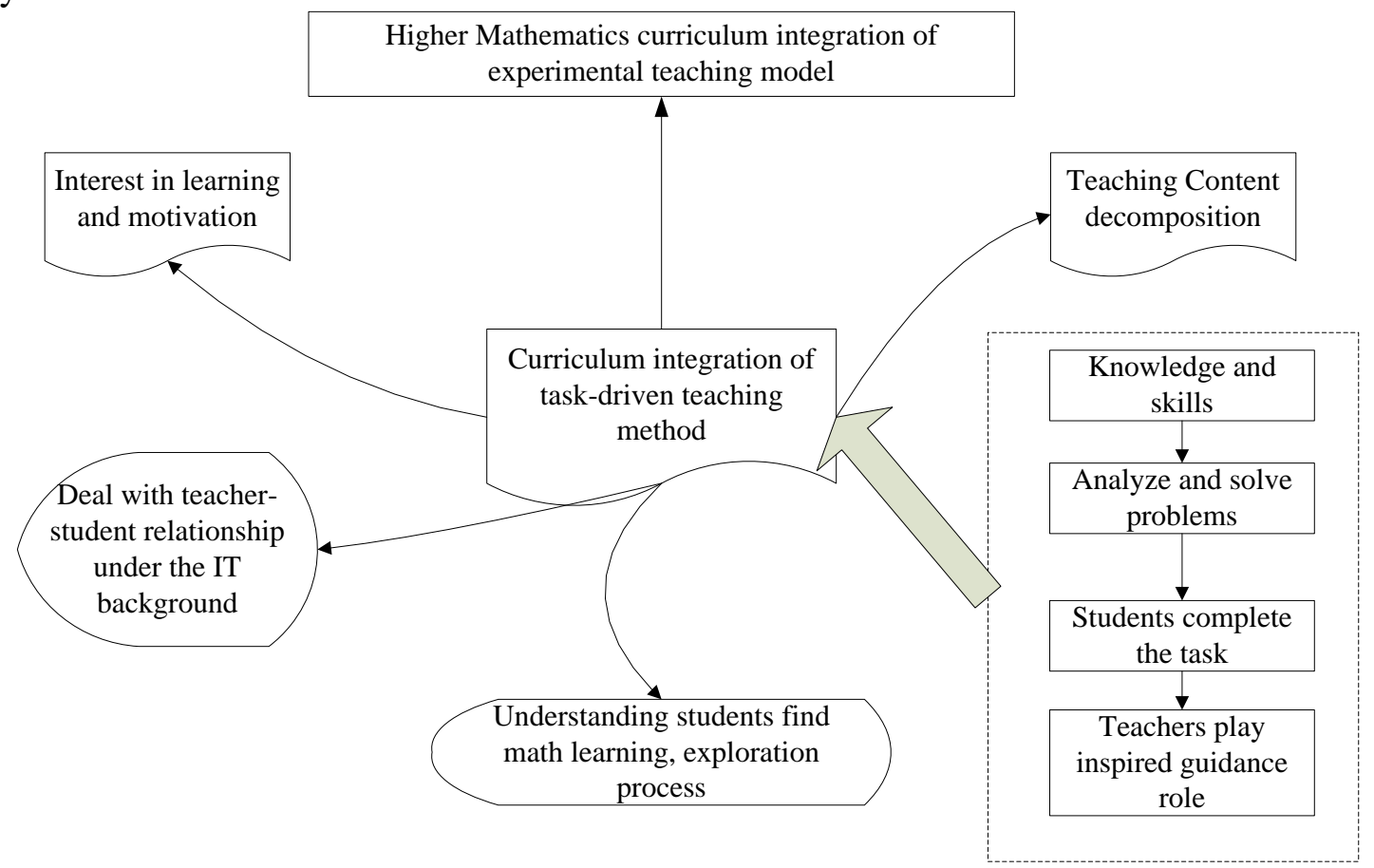

Diagram 1 Design model of computer platform of higher mathematics 
Teachers should combine with characteristics of higher mathematics curriculum, cultivate the discovery and exploration process for students to know mathematics learning, they should also use information technology to present and stress exploration, application and transfer of mathematics knowledge. In order to meet modern teaching requirements, teachers should combine with traditional teaching and information technology, try hard to make curriculum deign with higher level and higher quality as well as continually implement, revise and improve it, making information technology really have effect of promoting teaching, teachers directly teach their knowledge is the primary task of class. Introduction of information technology makes connotation between teachers and students have slight change, except for in the class experimental teaching model of centralized on teachers, students may obtain knowledge outside class, expand knowledge range, shorten knowledge gap between teachers and students by using information technology to make exploration, at the same time it is also changing the dominant position of teachers in class.

\section{Teaching model of higher mathematics based on information technology}

Task-driven teaching method is the precondition for integration of information technology and higher mathematics curriculum; it puts forward one kind teaching model of information and teaching experiment on the basis of making research on integration of information technology and higher mathematics. Teaching experiment is one kind of new teaching measure and model of higher mathematics, enabling mathematics teaching develop from the pure model of teaching by teachers, listening and exercise by students to together use information technology as well as realizing the aim of teachers and students together participating in learning. The aim of mathematics experiment is to discover and understand the relatively abstract or complicated content in mathematics by experiment. As it is indicated by diagram 2, teaching model of mathematics experiment based on information technology makes research methods of mathematics develop into model of computer technology with thinking, which provides broad application prospect for mathematics and mathematics experiment. Introduction of mathematics experiment injects much energy for curriculum teaching of higher mathematics, it can give students one kind of complete teaching, enabling students to make experiment under instruction by teachers, which can greatly enhance curiosity of students, stimulate their desire of exploration and creation, making learning process of students change into the process of experiment on their own, observation and discovery, guess and demonstration, reasonable reasoning and physical design. Teaching model of mathematics experiment can be concluded as 5 parts: create situation-mathematics experiment-summary and guess-reasoning and demonstration-outcome exchange, its main content is as follows: problem start-problem exploration-problem solution-reconsideration and innovation, this kind of model becomes much more effective in aspect of expanding teaching and cultivating mathematics modeling ability of students/ 


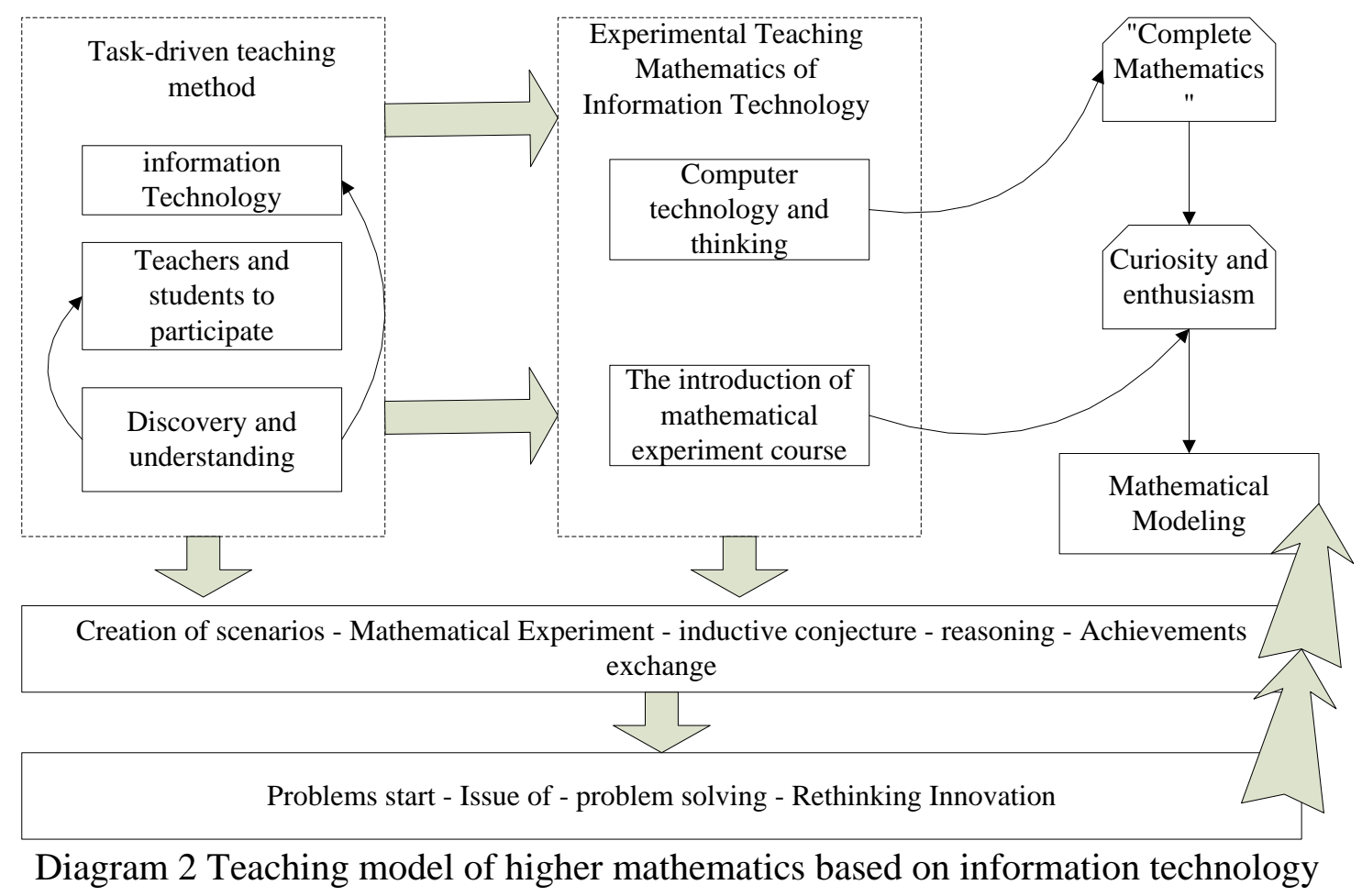

\section{Teaching model design of higher mathematics based on computer technology}

Computer-aided Instruction not only conforms to social constructivism, but also emphasizes creation and establishment of personal learning environment; it can actively construct its own cognition and knowledge through interaction with other people and social negotiation. We choose MySQL data base, MySQL is one kind of relation data base management system, relational data base stores data in the different tables while it dose not store all the data together, which increases speed and flexibility, SQ language of MySQL is the most usual standard language used to access data base. Dynamic website and storage of data base is coexisted, MySQL data base is the one universally used in PHP development, it adopts structured inquiry language to manage data, PHP dispatches and connects with MySQL data base function communicates with MySQL, PHP sends implemented operation to MySQL server, MySQL server sends the implemented command to PHP program.

On mathematics evaluation, the evaluation process generally confirms index of evaluating teachers according to real conditions of school, and then collects evaluation data of teachers according to index, the collected data base includes corresponding score of every index, and teachers develop work according to these sample data. From the collected data, it can be seen that evaluation data of each teacher includes $n$ indexes, it can regarded as vector of $n$ dimensions, it can be indicated as follows:

$$
X=\left(X_{1}, X_{2}, X_{3}, \cdots X_{n}\right)
$$

So as for the sample data of the whole teaching evaluation, it can indicated as follows: learning agent observes behavior and action of other agents and records it as $\pi\left(a^{j} \mid s\right)$, it is the prediction strategy of learning agent on companion agent $\mathrm{j}$, which means the probability of agent $\mathrm{j}$ implements action $a^{j}$ under the united state, obviously there is:

$$
\sum_{a^{j} \in A^{i}}\left(a^{j} \mid s\right)=1, \mathrm{j}=1,2, \ldots, \mathrm{I}-1, \mathrm{i}+1, \ldots, \mathrm{N}(2)
$$

In order to reduce computation complication and space storage, we adopt Robins-Monro method [7] to predict other agents. On time t, when agents observe implentation action $a_{t}^{j}\left(a_{t}^{j} \in A^{j}, j \neq i\right)$ of agent $\mathrm{j}$, we adopt the following ways to update its strategy. 


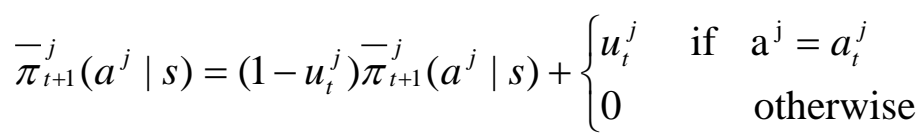

Of which, $\bar{\pi}_{t+1}^{j}\left(a^{j} \mid s\right)$ indicates the evaluated strategy renewal when agent $\mathrm{j}$ implements action on time $\mathrm{t}, u_{t}^{j}(0<\mathrm{u}<1)$ indicates the learning rate after updating, this sequence $u_{t}^{j}$ meets the following conditions:

$$
\sum_{t=0}^{\infty} u_{t}^{j}=\propto, \sum_{t=0}^{\infty}\left(u_{t}^{j}\right)^{2}<\propto(4)
$$

This paper adopts LIBSVM as experiment platform; the used training data and test data file form are as follows:

$$
<\text { labe1 }><\text { indexl }>,<\text { valuel }><\text { index } 2>\text {; }<\text { valte2 }>\text {... (6) }
$$

After using training sample data to make training on teaching evaluation system, teaching evaluation system will get one training model, model file contains some relevant parameters of SVM. The meaning of every parameter in the model is as follows:

-Parameters: it records stage value of parameter option in Libsvm_options

-nr_class: it indicates the category in the data set, which manes it is divided into how many kinds:

-totalSV: it represents the total number of support vector

-thoalSV: The contrast of constant term in decision function, $b$ in the decision function $w x+b$, that is $b=-r h o$.

-Label: classification of logo, which means the training label in teaching evaluation system, because it supports many kinds, so there are many integers.

-ProbA: Parameter A of pair probability information, it adopts parameter of SVM model output by probability, default will not be started.

-ProbB: parameter B in pair probability information

-nSV: The number of support vector in every category

-svcoef: it is one matrix, which loads coefficient of support vectors in decision function

-SVS: The obtained support vector of teaching evaluation system through training

\section{Practice analysis of higher mathematics}

Network environment not only guarantees autonomous learning of students becomes true, it also has unique function in aspects of research learning and cooperative learning, for example, in the solution of progression, differential equation and matrix, we can use computer and relevant software to make research on learning. .Under the environment of network teaching platform, students change from passive knowledge acceptance to active knowledge acceptance, they have learned the usual computer software and teaching software, they can actively learn knowledge, actively make exploration, actively cooperate and communicate. Through teaching management platform, it can also enrich mathematics culture and knowledge of students. This system except to collect the usual software of mathematics in teaching platform, but also introduces background and culture knowledge such as mathematics scientists, which can encourage learning passion of students. Take example of using Newton iterative method to solve nonlinear equations with higher comprehension and important learning content in higher mathematics, it exposes the mathematics experiment teaching model after integration between information technology and higher mathematics proposed by this paper.

Suppooe $y=f(x)$ is nonlinear function, it uses computer to solve equation $f(x)=0$, its basic idea is to gradually make nonlinear function $\mathrm{f}(\mathrm{x})$ linearization, so it approximately transfers nonlinear equation $f(x)=0$ into linear equation, this algorithm is the indication of using straight line to replace curve, using linear to replace nonlinear.

Suppose: function has continual 2-step derived function in range [a, b], of which a and b are 2 extreme points of range, and they meet $f(a) * f(b)<0 . f(x) f(b) \neq 0$ 
The derivation process of original algorithm includes plenty of mathematics analysis and theory knowledge, such as existence theorem for roots, meanings of differential coefficient and second derivative, Taylor's theorem etc, these relatively abstract knowledge combines with practice, which can make students experience importance of theory knowledge, at the same time it deepens their comprehension on these knowledge, and cooperates with multi-media dynamic presentation on teaching, which can make students lively understand principle of Newton iteration method.

1. input initial approximation $\mathrm{x} 0$ of root and allowable deviation

2.calculate $x_{n}+1 \rightarrow x_{n}-\frac{f\left(x_{n}\right)}{f^{1}\left(x_{n}\right)}$

3.Juege if $\left|x_{n+1}-x_{n}\right| \geq \varepsilon$, then $\mathrm{n} \rightarrow \mathrm{n}+1$,, otherwise, output $\mathrm{x}_{\mathrm{n}+1}$, and end.

the computer programming of algorithm is the important content of realizing this topic, in the following it will give Matlab source program of Newton iteration method

function $\mathrm{w}=$ newton(a,e)\%e is precision requirement, a is initial value.

$\mathrm{x}(1)=\mathrm{a} ; \mathrm{n}=\mathrm{l} ; \%$ initial values is given to $\mathrm{x}$

$x(2)=x(1)-f(x(1)) / g(x(1)) ; . \%$ is calculated by the 1 st initial value to one approximate $x 2$

whileabs $(x(n+1)-x(n))>e \%$ whileabs $(x(n+1)-x(n))>e \%$, it is judged by precision whether cycle is stopped or not

$\mathrm{n}=\mathrm{n}+1 ; \mathrm{x}(\mathrm{n}+1)=\mathrm{x}(\mathrm{n})-\mathrm{f}(\mathrm{x}(\mathrm{n})) / \mathrm{g}(\mathrm{x}(\mathrm{n})) ; \%$, Newton iterative formula

end

$\mathrm{x}(\mathrm{n}+1) \%$ output result

$\mathrm{n} \%$, the used iterative step

In order to better learn about effect of information technology and curriculum integration, we make step test on 2 classes in the test of peer group, we adopt methods of school pre-term test, mid-term test and final test, of which the pre-term test mainly investigates the basic knowledge and problem-solving skills of mathematics in high school for students, mid-term test is to test learning of the front half term of higher mathematics after students entering university, and final test is mainly tests degree of mastering knowledge and application after higher mathematics learning in the first semester is ended. According to test results, writer makes statistics and analysis on 3 times of class test results and the number of students who failed in 3 tests, of which, table 1 gives data statistics of class average result for 3 times, table 2 gives the distribution condition for those who failed in 3 tests.

Table 1 Statistics table of average result for class test

\begin{tabular}{ccc}
\hline test class & class A( ordinary group) & Class B(experiment group) \\
\hline pre-term & 72.2 & 72.2 \\
mid-term & 75.8 & 77.5 \\
final term & 75.2 & 79.6 \\
\hline
\end{tabular}

Table 2 Statistics table of students failed in class test

\begin{tabular}{ccc}
\hline Test class & Class A(ordinary group) & Class B(experiment group) \\
\hline pre-term & 5 & 5 \\
mid-term & 3 & 2 \\
final term & 1 & 0 \\
\hline
\end{tabular}

We use 2 learning classes of higher mathematics curriculum as investigation objects, we use anonymity way to make questionnaire survey, 2 classes totally distribute 130 pieces of questionnaire survey, each class has 65 pieces, of which, class A collects 64 pieces and class B collects 65 pieces, in the collected questionnaire survey, we make systematic statistics and profound analysis on 33 pieces randomly collected. The investigation indicates that class A and class B respectively have $67 \%$ and $83 \%$ students agree and very agree the importance of mathematics knowledge, students have common understanding on importance of mathematics, but in the learning of mathematics, class $\mathrm{A}$ and class B respectively have $19 \%$ and $31 \%$ students like mathematics class, $29 \%$ and $42 \%$ students are very like mathematics class. There are respectively $16 \%$ and $47 \%$ students in class A and class B 
actively learn mathematics, and $24 \%$ and $37 \%$ students are very actively learn mathematics. After learning higher mathematics, the enhancement condition on mathematics result by students, there are respectively $31 \%$ and $48 \%$ students in class A and class B think they have enhancement, and there are $18 \%$ and $39 \%$ students think they have big enhancement, while in class A there are $21 \%$ studnets think they have no enhancement.

From the above tables and results of questionnaire survey, we can see that students in class B are rather like mathematics class than students in class A, they have higher initiative to learn mathematics, so they have quick enhancement in mathematics, the teaching model of computer technology and higher mathematics curriculum integration are superior to the traditional teaching model in aspect of enhancing mathematics results of students.

\section{Summary}

This paper adopts teaching model of experiment teaching model, which improves teaching way, solves difficult points of teaching, students are easily to accept new knowledge, class teaching becomes much more rich and class learning atmosphere becomes much more relaxed, it increases class teaching quality, at the same time, this network teaching platform and multi-media software provide better communication condition, discussion environment for students, they use computer as tool and mathematics as method to make aided instruction on higher mathematics, enriches teaching resources, accelerates integration of information technology and mathematics teaching, increases teaching quality and efficiency, so it is one kind of helpful complementary for traditional mathematics teaching, it is worthwhile for use to make deep research and popularization.

\section{References}

[1]Zhang Hongyan. Discusison on Constructivism Teaching from Perspective of Model Structure. ModernEducation Technology,Vol17, 2007, p13-15..

[2]Zhang Xiaozhen. Discusison on Curriculum and Teaching Reform under Information Technology-from CAI to e-Learning. Journal of Southwestern Normal University: nature and science version,Vol27 (2) , 2002,p285-289.

[3]Wang Fucheng. Automatic Learning Application of University Students Based on Cloud Computing. Scientific Innovation Herald 2011.

[4] Liu Guannan. Research on Learning Environment Integration in Cloud Computing Times. Master's thesis 2010 , 2010.

[5] Liao Rongsu, Lai Xin, Liu Song. Considerations on Teaching Quality Evaluation of University Teachers. Forestry Education in China,Vol 19(4) ,2005,p 254-257

[6] Wu Tong, Wang Xiukun. Research on Teaching Quality Analysis and Evaluation Model Based on BP Neural Network. Microcomputer Information,Vol 4(09),2009,p 56一59. 